\title{
Wolf-Rayet stars in Local Group starbursts
}

\author{
Laurent Drissen \\ Département de Physique, Université Laval, \\ Sainte-Foy, QC G1K 7P4, Canada
}

Abstract. I describe recent spectroscopic observations of Wolf-Rayet stars in three giant H II regions of M 33 and in the Local Group starburst galaxy IC 10.

\section{Introduction}

Among massive stars, those of the Wolf-Rayet (WR) type are by far the easiest to detect and classify. Their broad and strong emission lines make them stand out of the crowd, even in dense regions such as the compact star clusters that ionize giant $\mathrm{H}$ II regions and starburst galaxies. Thanks to surveys in Local Group galaxies (Massey, these Proceedings) and improvements in theoretical models, the evolutionary status of Wolf-Rayet stars are now understood well enough to use them as diagnostics to infer the history of starburst regions. In unresolved clusters or starburst knots of distant galaxies (for which the metallicity is inferred from the nebular lines), the equivalent width of the 'WR-bumps' are good indicators of the age and upper mass limit of the stellar population (Schaerer, these Proceedings). Nearby starbursts offer an interesting testbed to understand the more distant, unresolved objects: individual stars can be counted and spectroscopically classified, allowing a direct comparison with the modelisation of the spatially integrated properties of their host cluster (see Vacca et al. 1995 for such a comparison in 30 Doradus).

The best way to unambiguously determine the WR content of dense clusters is to compare high resolution, high signal-to-noise 'on-line' (centered at $4686 \AA$ ) and 'off-line' images, using image subtraction. This technique has now been successfuly applied to clusters as far as 3.5 Mpc (Drissen et al. 1999), and could be pushed to $10 \mathrm{Mpc}$ with the use of adaptive optics on $10 \mathrm{~m}$-class telescopes. Because the flux and equivalent width of the $\mathrm{CIII} / \mathrm{C}$ IV $/ \mathrm{He}$ II blend at $\sim 4660 \AA$ in WC stars are generally much larger than that of the He II and N III/IV/V lines in WN stars in the same spectral region, imaging surveys naturally favor the detection of WC stars. A more thorough investigation, therefore, requires follow-up spectroscopy of the WR candidates to assess the completeness level, to classify them, and to identify stars with particularly interesting spectral features.

We present here some recent work on the WR population of two Local Group galaxies, M33 and IC 10. While M 33 is a 'quiet' spiral galaxy with a modest star formation rate, IC 10 is a much more active irregular galaxy, host of a widespread starburst. 

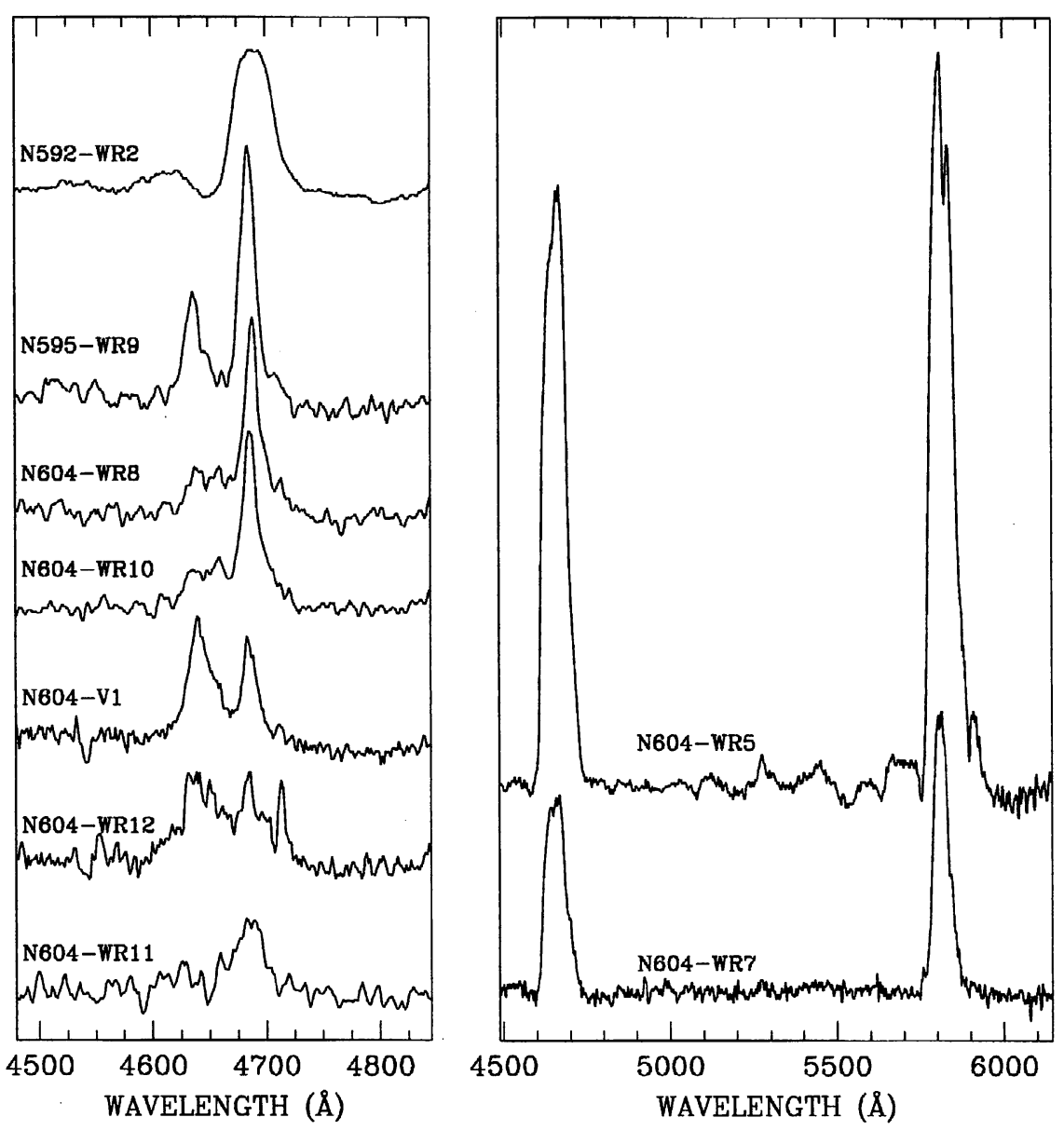

Figure 1. CFHT-MOS spectra of recently confirmed Wolf-Rayet and transition-type stars in NGC 604 and NGC 595. Left: the WN stars. Right: the WC stars. Note that the scales are different in the two panels. The strongest nebular lines have been removed to avoid confusion.

\section{2. $\quad$ M 33}

Drissen et al. $(1990,1993)$ detected WR candidates in the most luminous giant H II regions in M 33, namely NGC 592, NGC 595 and NGC604. Some of them were already known and spectroscopically classified, but good spectra of the faintest candidates and those with weak equivalent width were still lacking. We have therefore obtained, in 2000 August and 2001 October, CFHT-MOs spectra of most WR stars in these HII regions. Some of these spectra are shown in Figure 1. All WR candidates show He II $\lambda 4686$ in emission, which testifies to the efficiency of the imaging technique. We are now confident that the completeness level of the WR survey in these clusters is very high, since some of the WR candidates are in fact transition-type stars (Of/WNL) with equivalent width of 
$\sim 10 \AA$. Only the stars in regions of high extinction could have been missed. Recent narrow-band nebular imagery of these clusters are presented by Bosch et al. (2002).

\subsection{NGC 604}

NGC 604 is one of the most scrutinized giant H II region (see González Delgado \& Pérez 2000) for a recent compilation of the literature on this object). It is second only to 30 Doradus in the Local Group in terms of $\mathrm{H} \alpha$ luminosity and number of massive stars. It has been known for a long time to harbour WR stars: its global spectrum, as well as most randomly oriented long-slit spectra show the WR-bumps, but spectroscopy of individual stars are rare. The spatial distribution of WR stars is very similar to that of the other bright stars, suggesting that the age spread between the sub-clusters was small. This is confirmed by González Delgado \& Pérez (2000) who conclude from the analysis of HSTWFPC2 images and IUE spectra that the burst occured instantaneously $3 \mathrm{Myr}$ ago. A dozen WR candidates are known in the region (Drissen et al. 1993). Half of them have a rather small excess in the 'on-line' $\lambda 4686$ filter, and spectroscopy was required to confirm them as genuine WR stars.

(i) NGC604-WR8 and NGC604-WR10 are spectroscopically very similar, in terms of line ratios and equivalent width, to WR 25 (HD 93162, WN6h) in the Carina nebula (see figure 1 in Walborn \& Fitzpatrick 2000).

(ii) NGC604-V1 is particularly interesting. It was named V1 because of its photometric variability (by more than 0.5 mag within a single night; Drissen et al. 1990), but a comparison of spectra obtained in 1992 and 2001, with the same instrument, also shows a variation in the relative strength of the He II $\lambda 4686$ and N III $\lambda \lambda 4634,41$ emission lines. The other He II lines at $4541 \AA$ and $5411 \AA$ are in absorption. He I lines show P-Cygni profiles, but the very strong nebular components are very difficult to remove and could be responsible for a large part of the emission component.

(iii) NGC604-WR7 and NGC604-WR11 are located in a region where the absorption is quite high ( $A_{V} \simeq 3 \mathrm{mag}$ ), which explains their faintness in the visible and the weak emission line flux seen in imagery; near infrared images of NGC 604 (Plante, private communication) suggest the presence of heated dust in this area. (iv) The spectrum of NGC 604-WR12 is quite peculiar, with N III stronger than He II. The small equivalent width of these lines suggest that it is a transition Of/WN star.

We have confirmed that NGC 604 contains a dozen WR stars, including three WC stars. This number is consistent with starburst models based on a young single burst (González Delgado \& Pérez 2000).

\subsection{NGC 595}

NGC 595 is about three times less luminous and massive as NGC604, but it harbours a similar number of WR stars. This is rather puzzling, especially if one considers that only one of them is of WC subtype (NGC595-WR3), which our recent spectrum (not shown in Figure 1) classifies as WC5. Malumuth et al. (1996) derive an age of 4.5 Myr for NGC 595, based on HST-WFPC2 images alone, but a detailed investigation of its integrated properties remains to be done. We show in Figure 1 the spectrum of NGC595-WR9, a WN7 star, which looks very similar to that of the other WN stars in this cluster. 


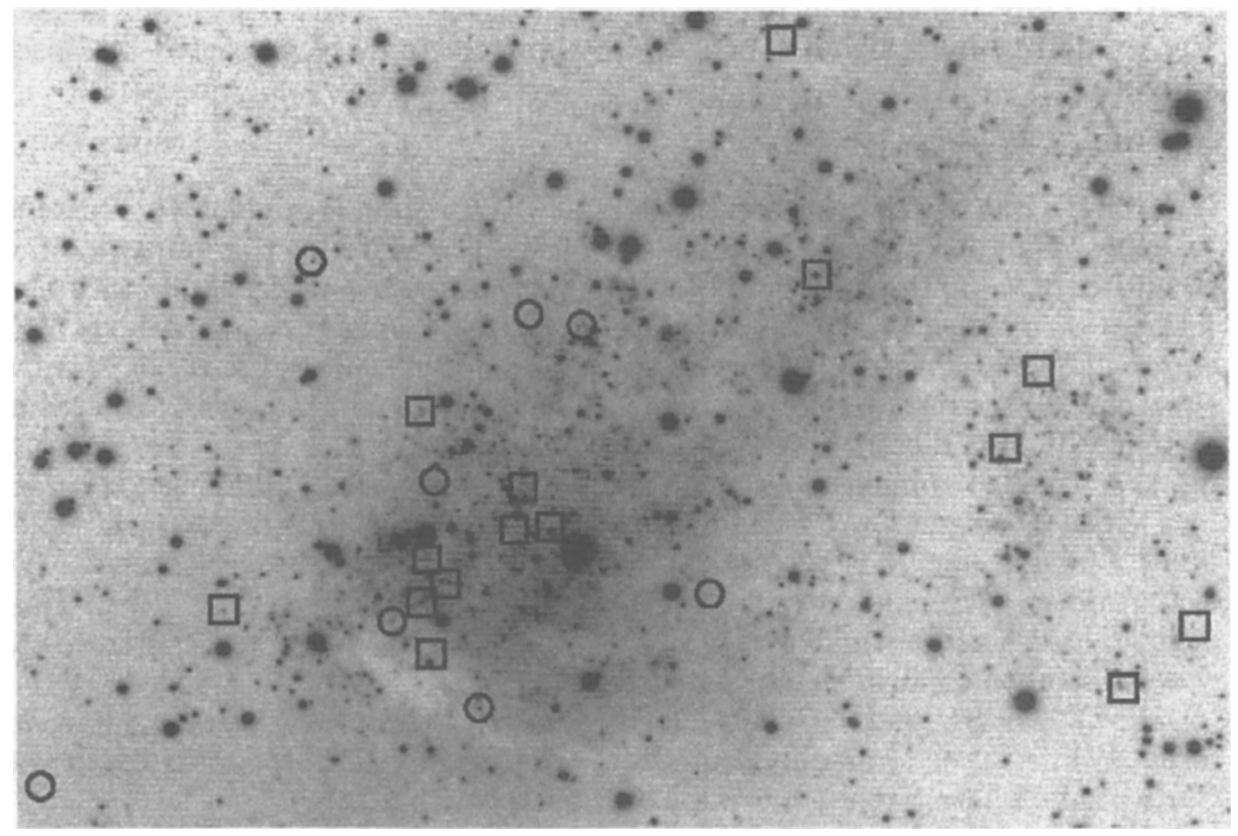

Figure 2. Location of all known confirmed Wolf-Rayet stars in IC 10, shown here on a Gemini-North $V$-band image (about $5^{\prime} \times 3^{\prime}$, with North to the top and East to the left). WN stars are identified as circles, WC stars as squares. The distribution of WR stars follows that of the other stars in the galaxy.

\subsection{NGC 592}

Only two WR stars are known in NGC 592, which is a factor of 12 times less luminous than NGC 604: the WNL star NGC 592-WR1 and, in the heart of the H II region, NGC592-WR2 which was discovered by Drissen et al. (1990) but never spectroscopically confirmed until now. Its spectrum was obtained with a very wide $\left(5^{\prime \prime}\right)$ slit, at high airmass and at the very end of the observing run. It shows strong NV lines and broad He II lines at 4686 and $5411 \AA$ typical of WN3-4 stars, a spectral type which is uncommon in giant $\mathrm{H}$ II regions.

\section{IC 10}

The low-mass IBm galaxy IC 10 is remarkable in the Local Group for its very high star formation rate: its large far-IR luminosity (Mellise \& Israel 1994), non-thermal radio continuum (Yang \& Skillman 1993) and high surface density of massive stars (Massey \& Armandroff 1995) are direct evidences of a young, wide-scale burst of star formation. Despite the fact that, at a distance of about $1 \mathrm{Mpc}$, IC 10 can be considered as the nearest example of a blue compact dwarf galaxy (Richer et al. 2001), its stellar content has been investigated only during the past decade. This is at least in part due to high obscuration $\left(A_{B} \simeq 3.5 \mathrm{mag}\right)$ by Milky Way dust (IC 10 lies only $3^{\circ}$ below the Galactic plane). The starburst phenomenon in IC 10 is also reflected by its large population of WR stars: 15 
WR stars are spectroscopically confirmed (Massey \& Armandroff 1995) and 13 new candidates have recently been discovered by narrow-band imagery (Royer et al. 2001). Not only is this large WR population remarkable for such a low metallicity galaxy $\left(Z=0.15 \mathrm{Z}_{\odot}\right.$; Richer et al. 2001$)$, but the $\mathrm{WC} / \mathrm{WN}$ ratio is much higher than anywhere else in the Local Group.

The observations were obtained in 2001 December and 2002 January with the newly commissioned GMOS multi-object spectrograph (Crampton et al. 2000) attached to the $8.1 \mathrm{~m}$ Gemini-North telescope. Two fields were necessary to cover the extent of the galaxy, with the B600 grating. The GMOS spectra will be shown elsewhere, but they confirm that most of the WR candidates from Royer et al. (2001) are indeed genuine WR stars, with the noticeable exception of the WC9 candidates. The WC/WN number ratio thus remains very high, about 2 (see also Massey, these Proceedings).

The average $V$ magnitude of the confirmed WR stars in IC 10 is $22.0 \mathrm{mag}$; assuming a distance of $0.9-1.0 \mathrm{Mpc}$ and a uniform foreground exctinction of $A_{V}=2.45$, this corresponds to $M_{V} \simeq-5.2$ to -5.4 . This is $0.6-0.9$ mag brighter than the average $M_{V}$ of WR stars in the LMC (Breysacher et al. 1999) or the SMC (Massey \& Duffy 2001). The highly variable internal exctinction across IC 10 makes matter even worse, so it is therefore very likely that a large number of WR stars remain to be found in this galaxy. A deeper survey in IC 10 will certainly detect a large number of WR stars, most likely of WN subtype. Indeed, Holmes \& Massey (2002) claim the discovery, via imagery, of at least a dozen WR candidates, and possibly up to 60 .

Despite the obvious incompleteness of the current surveys, Figure 2, which shows the location of all 23 spectroscopically confirmed WR stars in IC 10, confirms the claim by Hunter (2001), that the recent starburst is really widespread: it is obvious that WR stars are spread over a large surface of the galaxy, showing no preference for specific clusters of tight $O B$ associations.

Acknowledgments. I would like to thank my collaborators on this project, in particular Paul Crowther, Luc Dessart and Pierre Martin, as well as JeanRené Roy and Inger Jorgensen who obtained the data with the Gemini-North telescope.

\section{References}

Bosch, G., Terlevich, E., Terlevich, R. 2002, MNRAS 329, 481

Breysacher, J., Azzopardi, M. Testor, G. 1999, A\&AS 137, 117

Crampton, D., Fletcher, J., Murray, J.I., et al. 2000, in: M. Iye \& A.F. Moorwood (eds.), Optical and IR Telescope Instrumentation and Detectors, SPIE 4008, 114

Drissen, L., Moffat, A.F.J., Shara, M.M. 1990, ApJ 364, 496

Drissen, L., Moffat, A.F.J., Shara, M.M. 1993, AJ 105, 1400

Drissen, L., Roy, J.-R., Moffat, A.F.J., Shara, M.M. 1999, AJ 117, 1249

González Delgado, R.M., Pérez, E. 2000, MNRAS 317, 64

Holmes, S.B., Massey, P. 2002, AAS 200, 4609

Hunter, D.A. 2001, ApJ 559, 225

Malumuth, E.M., Waller, W.H., Parker, J.Wm. 1996, AJ 111, 1128

Massey, P., Armandroff, T.E. 1995, AJ 109, 2470

Massey, P., Duffy, A.S. 2001, ApJ 550, 713 
Melisse, J.P.M., Israel, F.P. 1994, A\&A 285, 51

Richer, M.G., Bullejos, A., Borissova, J., et al. 2001, A\&A 370, 34

Royer, P., Smartt, S.J., Manfroid, J., Vreux J.-M., 2001, A\&A (Letters) 366, L1

Vacca, W.D., Robert, C., Leitherer, C., Conti, P.S. 1995, ApJ 444, 647

Walborn, N.R., Fitzpatrick, E.L. 2000, PASP 112, 50

Yang, H., Skillman, E.D. 1993, AJ 106, 1448

\section{Discussion}

MÁrChenko: You have shown a picture with the large spread of the C III and C IV line intensities for WC stars in IC 10. Do you think it is intrinsic? Can it be, at least partially, caused by crowding?

DRISSEN: Single WC stars show a spread in their C IV equivalent widths, but it is very likely that a significant part of the spread we see in IC 10 could be due to crowding and/or binarity.

MASSEY: Very nice work! I just wanted to clear up one point - the stars in M33 outside of your giant H II regions are not all 'field' - many of them are found in M 33's OB associations, although some are true field.

DRISSEN: Thank you. Indeed, by 'field' stars I meant those outside of giant H II regions.

Koenigsberger: So, let me get this straight: How may extragalactic WR's are found in clusters vs. being field stars?

DRISSEN: I do not have the numbers in mind. In M 33, about $20 \%$ of the known WR stars are found in the few giant H II regions (NGC 604, NGC 595, NGC 592 and NGC 588). This number is likely to go down, since the completeness level is higher in giant $\mathrm{H}$ II regions.

Johnson: This is a question that Phil Massey posed to me four years ago at IAU Symposium No. 193 in Puerto Vallarta, and it has nagged me ever since. As you pointed out, IC 10 has widespread star formation, but no compact star clusters. Do you have any theories about why this might be? I think that if we can figure out the reason for this, it will give us some incredible physical insight into cluster formation.

DRISSEN: An H I map of IC 10 shows a huge, elongated H I cloud that extends over five times the diameter of the visible galaxy, and is probably infalling. This might be the cause of the present burst. Now, why does it result in a widespread star formation instead of the formation of super star clusters (like in NGC 1569 for instance), I don't know.

WALBORN [comment on question by Johnson]: It is interesting to turn the question around and ask why the LMC, for instance, has massive compact clusters. Their formation may require very massive molecular clouds with high relative densities. In the LMC these may be caused by interactions with the SMC and the Galaxy, conditions which may not occur in IC 10.

MASSEY [comment on question by Johnson and on comment by Walborn]: Deidre Hunter has noted that within the LMC Constellation III provides a fascinating contrast to the 30 Dor/R 136 complex. Both probably had a similar amount of star formation, but in one case (R 136) it was in the form of a super star cluster, and in the other case it 
was just a big, rich OB association. Why? Deidre speculated that maybe the bar has something to do with it.

MAEDER: If the starbursts are almost instantaneous, the WN/WC ratios are varying as a function of age, since the O-type stars firstly enter the WN and then the WC stage, would it be an effect intervening in very localized regions like NGC 604 and NGC 595, or even to some extent in IC 10 ?

DRISSEN: I think it is reasonable to assume that the star formation in compact clusters such as R 136 was instantaneous. In regions like NGC 604, which are more like massive $\mathrm{OB}$ associations with diameters of $\sim 50 \mathrm{pc}$, the burst could have been longer $(\Delta t \approx 1-2$ $\mathrm{Myr}$ ?). The WC/WN ratio is therefore not only a function of the burst age, but also of the burst duration. In the case of IC 10, we can safely assume a continuous star formation rate.

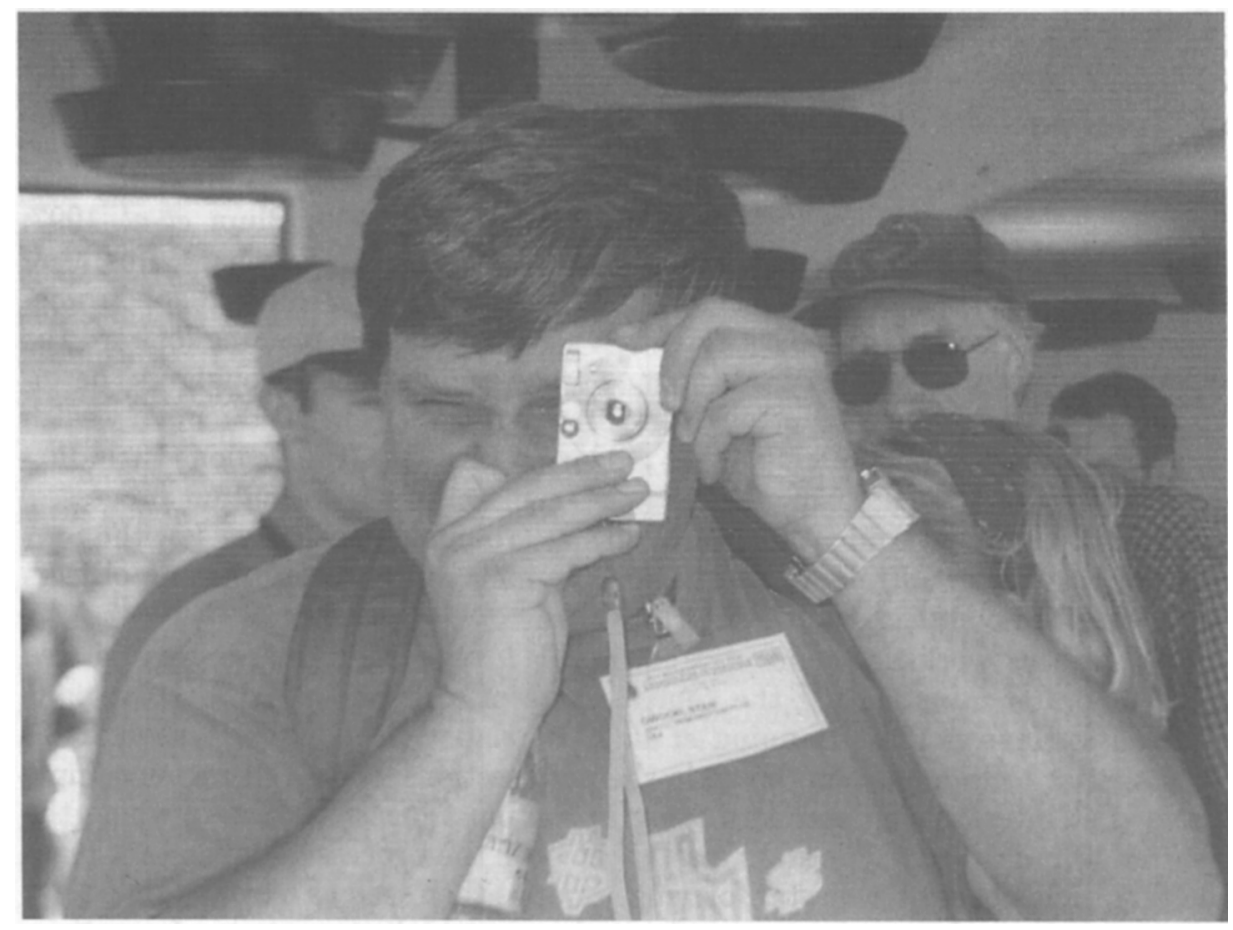

Stannie sure-shot 